\title{
Germanica
}

\section{Kassiber schreiben. Rainer René Muellers Verdichtungen}

Chiara Caradonna: Écrire des messages secrets. La poétique de la condensation chez R.R. Mueller

Chiara Caradonna: Writing secret messages. Rainer René Mueller's condensations

\section{Chiara Caradonna}

\section{OpenEdition}

\section{Journals}

Édition électronique

URL : http://journals.openedition.org/germanica/6493

DOI : 10.4000/germanica.6493

ISSN : 2107-0784

\section{Éditeur}

Université de Lille

\section{Édition imprimée}

Date de publication : 26 juin 2019

Pagination : $17-40$

ISBN : 978-2-913857-42-1

ISSN : 0984-2632

\section{Référence électronique}

Chiara Caradonna, „Kassiber schreiben. Rainer René Muellers Verdichtungen“, Germanica [Online], 64 |

2e trimestre 2019, Online erschienen am: 01 Januar 2021, abgerufen am 25 Februar 2021. URL: http://journals.openedition.org/germanica/6493 ; DOl: https://doi.org/10.4000/germanica.6493 
Voix de poètes 



\title{
Kassiber schreiben. Rainer René Muellers Verdichtungen
}

\author{
Chiara Caradonna \\ The Martin Buber Society of Fellows \\ in the Humanities and Social Sciences \\ The Hebrew University of Jerusalem
}

(gegen jede Gegenwart immer quer schreiben) ${ }^{1}$

Es war meine Behauptung, das lyrische Gebilde sei stets auch der subjektive Ausdruck eines gesellschaftlichen Antagonismus ${ }^{2}$.

\section{Ein Umschlag}

Auf dem schwarzen Umschlag von Rainer René Muellers erstem, längst vergriffenem Gedichtband LiedDeutsch, der 1981 im kleinen Verlag Desire \& Gegenrealismus erschien ${ }^{3}$, ist eine Manuskriptseite aus

1. — Rainer René Mueller, „Märzlich“, in: Ders., LiedDeutsch, Heidelberg, Verlag Desire \& Gegenrealismus, 1981, S. 17.

2. - Theodor W. Adorno, „Rede über Lyrik und Gesellschaft“, in: Ders., Noten zur Literatur, Frankfurt a.M., Suhrkamp, 1974, S. 58.

3. - Bereits 1982 beschwerte sich der Literaturkritiker Uwe-Michael Gutzschhahn über die mangelnde Aufmerksamkeit, die Kleinverlagen entgegengebracht würde, was zum Untergehen wichtiger Stimmen, darunter Muellers, geführt habe (vgl. „Rainer 
Friedrich Hölderlins Nachlass abgedruckt. Das Bild ist stark verkleinert, die Schrift kaum lesbar. In der oberen linken Ecke ist ein einzelnes, vereinsamtes knappes Wort $\mathrm{zu}$ sehen, dann folgt ein Weißraum, anschließend eine lange Reihe kurzer Verse. Erst die Bildbeschreibung auf der Rückseite des Titelblatts verrät, um welche Manuskriptseite es sich handelt. Das Motto, das den Gedichten vorangestellt ist, bietet die Transkription von zwei Zeilen der Handschrift sowie ihre Zuordnung zu einem bestimmten hymnischen Entwurf:

der Fels ist zu Weide gut, das Trockene zu Trank.

Hölderlin, „Der Adler“

1975 war im neu gegründeten Frankfurter Verlag Roter Stern der Einleitungsband zur Frankfurter Hölderlin-Ausgabe, herausgegeben von D. E. Sattler, veröffentlicht worden. Unter anderem anhand des Entwurfs von „Der Adler“ wurde dort die neue, revolutionäre Vorgehensweise illustriert, mit der in den darauffolgenden Jahren Hölderlins Werk dem breiteren Publikum vorgelegt werden sollte 4 . Die Übernahme einer der Seiten als Umschlagbild für LiedDeutsch bezeugt die unmittelbare Rezeption dieser neuen Ausgabe, die sonst im Buch keine ausdrückliche Erwähnung findet.

In der Wahl des Bildes bzw. des im Verhältnis zum Titel paradoxen Hölderlin-Zitats sind in nuce Motive angelegt, die nicht nur für LiedDeutsch, sondern für Muellers gesamtes lyrisches Schaffen bis hin zur neuesten Sammlung geschriebes. selbst mit stein (Heidelberg, Edition aouey, 2018) von grundlegender Bedeutung sind. Drei dieser Motive werde ich (unter Verzicht auf viele andere) im Folgenden aufgreifen, um erstmalig eine Übersicht von Muellers Werk zu bieten, das - im Zeichen einer staunenswerten Strenge und Kohärenz, und doch ohne Erstarrung - einen zeitlichen Rahmen von mehr als vier Jahrzehnten deckt.

René Mueller. Lieddeutsch, querliegend“, Deutsches Allgemeines Sonntagsblatt, 5/1982, S. 20). Wenn auch die Gründe nicht ausschließlich - wie Gutzschhahn nahelegt - bei der marktwirtschaftlichen Ausrichtung des deutschsprachigen Literaturbetriebs zu suchen sind, so soll dies in Bezug auf Muellers Werk lange Zeit - zumindest bis zu dessen Wiederentdeckung seit 2015 - wahr bleiben. Ich möchte von Herzen Rainer René Muellers langjährigem Freund Rainer Blum danken, der mir sein Archiv zur Verfügung gestellt hat. Dort sind nicht nur Muellers verstreute Veröffentlichungen in Zeitschrift- und Buchformat beinah vollständig aufbewahrt, sondern es liegen auch frühe Gedichte und Prosa (Typoskripte), Rezensionen, Bildmaterial sowie zu diesem Zeitpunkt noch unerschlossene Briefe vor. Aus diesem Fundus wird im Folgenden mit der Angabe „Archiv Rainer Blum, Berlin“ zitiert.

4. - Vgl. Friedrich Hölderlin, Sämtliche Werke. Frankfurter Ausgabe. Einleitungsband, hrsg. von D. E. Sattler, Frankfurt a.M., Stroemfeld, 1975, S. 41-50. Das Zitat unterscheidet sich vom Abdruck in Sattlers Ausgabe durch die Kleinschreibung der Anfangsbuchstaben sowie durch die Schreibung „Weide“ statt „Waide“. 
Die Hälfte davon hat Mueller nur zurückhaltend am deutschsprachigen Literaturbetrieb teilgenommen. Anfang der achtziger Jahre galt Mueller, der das Erbe von Paul Celan, Ernst Meister und Ernst Jandl mit Einflüssen aus der französischen und englischen poetischen Tradition verband, als neue aufkommende Stimme in der deutschsprachigen Lyrik. Seine Gedichte erschienen in zahlreichen Zeitungen, Sammelbänden und Zeitschriften, 1983 gewann er den Leonce-undLena-Förderpreis und war Stipendiat am Literarischen Colloquium Berlin. Mit den Bändern Kreuzungen/Sturzfigur und Strichscharen (1986) zog er sich bewusst aus dem Betrieb zurück, erst 1994 erschien der Band Schneejagd/Chasse de neige, dessen Veröffentlichung ursprünglich für das Jahr 1983 geplant worden war. Die Widmung an den langjährigen Freund Rainer Blum in einem Exemplar der Zeitschrift Park aus dem Jahre 1985, in der Drei Gedichte für keinen abgedruckt wurden, lässt ahnen, aus welchem Geiste heraus der Rücktritt aus der literarischen Szene stattfand: ,ich habe zwei Jahre lang / geschrieen. Nun schreibe / ich für keinen mehr; wozu / weiß ich nicht. Ich muß / mich wenigstens für das / Echo aufrechthalten, damit / es nichts zum Aufprall / hat." 5 . Nach der Veröffentlichung einiger weniger Texte in der Zeitschrift Zwischen den Zeilen (1996) wurde es dann ganz still um ihn, bis im Jahre 2015 der Dichter Dieter M. Gräf und der Verleger Urs Engeler die Erscheinung eines Sammelbandes mit bereits veröffentlichten sowie bis dahin noch unveröffentlichten Gedichten Muellers unter dem Titel poèmes - poëtra veranlassten. Es geschah ein literaturgeschichtlich eher seltenes Ereignis: ein vergessener Autor wurde noch zu Lebzeiten wiederentdeckt und gewann in kürzester Zeit viel Aufmerksamkeit sowie neue begeisterte Leser und Zuhörer6. Seit 2015 hat Mueller mit großem Erfolg seine Gedichte an zahlreichen Orten in Deutschland, Frankreich und Israel gelesen. Parallel dazu begann für ihn eine Zeit hoher Produktivität, die 2018 in die Veröffentlichung des bereits erwähnten Bandes geschriebes. selbst mit stein mündete. Damit kam nach langem Schweigen eine Stimme erneut zu Wort, die es nun im Rückblick erlaubt, die Entwicklung ihrer poetischen Sprache, vom Ende der siebziger Jahre bis heute, nachzuzeichnen.

Stichwortartig sind also die drei Motive, auf die ich eingehen werde: das Lied, die Herkunft (sowohl individuell als auch allgemein,

5. - Archiv Rainer Blum, Berlin.

6. - So gestand der Literaturkritiker Joachim Sartorius in der Frankfurter Allgemeinen Zeitung vom 15. Juli 2016: „Es war einer meiner großen Fehler, Rainer René Mueller nicht in Niemals eine Atempause, mein Handbuch der politischen Poesie im zwanzigsten Jahrhundert, aufzunehmen. Er hat dort Anrecht auf einen bevorzugten Platz - und nicht nur dort. [...] Wir müssen seine Gedichte lesen [...]. Die Entdeckungen sind ungeheuer und nehmen uns mit." Vgl. auch Stefan Ripplinger, ,Jäger im Schnee“, Neues Deutschland, 17. September 2016. 
im Sinne einer familiären wie auch poetischen Genealogie) und der Stein. Sie sind auf mehrfache Weise miteinander verwoben und jedes von ihnen ließe sich weit ausführen. Mit dem ersten, dem Lied, gehen Fragen nach dem Verhältnis von Kunst- und Volkslied, Gedicht und Gesang, Gesang und Schrift, Gedicht und Musik, allgemeiner nach dem gegenwärtigen Status des Lyrischen einher. Mit dem zweiten, der Herkunft, ist die traditionsreiche Frage nach der prekären Verortung der poetischen Stimme in Zeit und Raum und angesichts ihrer Vorgänger gestellt. Mit dem dritten Motiv, dem Stein, das die beiden vorherigen sinnbildlich mit einschließt, ist die Frage nach der poetischen Form im Verhältnis zur Zeit aufgeworfen. Ihnen gemeinsam ist der Angelpunkt, um den sich der Titel des ersten Bandes LiedDeutsch programmatisch dreht, nämlich die Fragestellung: Was ist deutsch? Das dritte Gedicht der Sammlung, „Lirum, larum“, will sie beantworten, da es mit dem Vers beginnt: „deutsch, das ist auch"7. Aufgrund der Betonung auf "auch“" erhebt die Definition keinen Anspruch auf Ausschließlichkeit. Sie versteht sich vielmehr als Fortsetzung und Ergänzung, wie es auch die Kleinschreibung des Anfangsbuchstabens - ein häufig auftretendes Phänomen in Muellers Lyrik - signalisiert. Ein jedes Gedicht ist immer auch Teil eines laufenden Gesprächs mit anderen Texten, innerhalb eines selben Bandes und überhaupt. Im jüngsten Band geschriebes beginnen siebzehn der fünfundzwanzig Gedichte mit Auslassungspunkten.

\section{deutsch, das ist auch}

Die Frage „Was ist deutsch?“ hat Tradition. Theodor W. Adorno ging 1965 in einem Radiobeitrag, der 1969 veröffentlicht wurde, bereits darauf ein. Gemäß der Doppeldeutigkeit der Formulierung beginnt Adorno zunächst mit der Bestimmung eines vermeintlich deutschen Nationalcharakters. Die Frage selbst bedarf der kritischen Reflexion, da sie leicht zur Stereotypie führe ${ }^{8}$. Adorno erinnert daran, dass Richard Wagner 1878 die Frage auf seine Weise beantwortete, indem er den deutschen Hang zur Innerlichkeit und die Abneigung des Deutschen gegenüber jeglichem Zweckdenken betonte. Wagners Schilderung deutschen Geistes ist unverhohlen antisemitisch. Die

7. - „Lirum, larum“ ist in den letzten Jahren zu neuer Berühmtheit aufgestiegen, vor allem dank der Besprechung von Joachim Sartorius in der Frankfurter Allgemeinen Zeitung vom 15. Juli 2016.

8. - Vgl. Theodor W. Adorno, „Auf die Frage: Was ist deutsch“, in: Ders., Kulturkritik und Gesellschaft II, Gesammelte Schriften, Bd. 10.2, hrsg. von Rolf Tiedemann, Frankfurt a.M., Suhrkamp, 1977, S. 691-701, hier: S. 692. Jüngst hat sich der Philosoph Peter Trawny erneut und an Adorno anknüpfend der Frage gewidmet, vgl. Peter Trawny, Was ist deutsch? Adornos verratenes Vermächtnis, Berlin, Matthes \& Seitz, 2016. 
Juden - so Wagner - würden die „liebenswürdige“ Unfähigkeit des Deutschen, „die Innigkeit und Reinheit seiner Anschauungen und Empfindungen“ zum „eigenen Vortheil“" umzusetzen, ausbeuten, um sich zu bereichern ${ }^{9}$. Wagner entwirft das Deutsche in Abgrenzung zum als Fremdkörper in der eigenen Gesellschaft vorgestellten Anderen. Adorno hebt daraufhin das Verhängnisvolle an Wagners Verständnis deutscher Identität hervor. Mit seiner sozialen Entsprechung im bürokratischen Pflichterfüllungsdenken habe es im 20. Jahrhundert vernichtende Folgen gezeitigt:

Geschichte erweist sich daran, bis heute, als Schuldzusammenhang, daß die höchsten Produktivkräfte, die obersten Manifestationen des Geistes verschworen sind mit dem Schlimmsten. Noch dem Um seiner selbst willen ist, im unerbittlich integern Mangel an Rücksicht auf den anderen, auch Inhumanität nicht fremd. [...] Wenn man etwas als spezifisch deutsch vermuten darf, dann ist es dies Ineinander des Großartigen, in keiner konventionell gesetzten Grenze sich Bescheidenden, mit dem Monströsen ${ }^{10}$.

Nach dieser Feststellung - deren Gehalt in Muellers Werk allgegenwärtig ist - geht Adorno dazu über, das Wort „deutsch“ nicht als adjektivische Bezeichnung der Nation, sondern als Sprache, in der er aufwuchs und zu der er nach dem Exil zurückkehren wollte, $\mathrm{zu}$ beschreiben. Adorno spricht von der „Wahlverwandschaft zur Philosophie“, die der deutschen Sprache eigen sei, aber auch davon, dass man sich als Muttersprachler einen Schwierigkeitsgrad in der eigenen sprachlichen Äußerung zutraut, vor dem man sich in einer sich später angeeigneten Sprache scheut. Die Präzision und Ausdruckskraft des Deutschen bergen jedoch, so Adorno, auch die Gefahr, eigene Aussagen ungenügend kritisch zu betrachten, im Vertrauen, die Sprache spreche für sich. Das aber führe zum „Jargon der Eigentlichkeit“11.

Dass sich der 1949 in Würzburg geborene Mueller gleich im ersten Gedichtband programmatisch mit dieser sperrigen Frage auseinandersetzte und den nicht geringen Anspruch erhob, ein „LiedDeutsch“ vorzulegen, könnte als Zeichen der Hybris gedeutet werden. Es kommt darin die Absicht zum Ausdruck, den von Adorno anvisierten kritischen Umgang mit der deutschen Sprache und ihrer Geschichte zu praktizieren. Sie ist zugleich auch eine politische Stellungnahme. Doch Mueller ist - das bezeugt eines seiner ersten veröffentlichten

9. — Richard Wagner, „Was ist deutsch?“, Bayreuther Blätter, 2/1878, S. 29-52, hier: S. 35.

10. - T. W. Adorno, „Auf die Frage: Was ist deutsch“, a.a.O., S. 694.

11. - Ebd. Kritik an Heideggers ,Jargon der Eigentlichkeit“ übt auch Mueller, insbesondere im jüngsten Band geschriebes. selbst mit stein. 
Gedichte - von Beginn an mit einer selbstbewussten poetischen Geste aufgetreten, die die philosophische Thematik vom Verhältnis zwischen Mensch und Sprache berührt:

Vom Hermetischen
ich will
euch Worte machen
bewohnbare auch
solche die ihr
anfassen könnt
nehmt sie mit
schaut euch um
Haus ist nicht
Haus
Baum ist nicht
Baum
ich sage euch
Baum ist Baum
und Haus ist
Haus
wohnt in euch
wohnt lange
die Dauer allein
gibt euch dann
auch die Worte
fürs Ganze

Es klingen hier sowohl Hölderlins „Was bleibet aber, stiften die Dichter"13 als auch Heideggers Wendung von der Sprache als Haus des Seins an ${ }^{14}$. Doch der ontologische Diskurs wird durch eine ständige Umkehrbewegung unterwandert, die, indem sie die Möglichkeiten des Versumbruchs ausschöpft, Abstraktion und Konkretheit stets ineinander über- und aufeinander zurückführt. Sollen etwa die vom sprechenden Ich zu schaffenden Worte anfangs durch ein Kollektiv (vermutlich der Leser) „,bewohnbar“ sein, so ist hingegen am Ende das Wort Haus, in dem man hätte wohnen sollen, beim Kollektiv zuhause („Haus / wohnt in euch“, V. 15f.). Zwar ist bei der Anschauung kein Haus (V. 8), wohl aber, wenn es ausgesprochen wird (V. 14). Zuletzt ist es - anders als angekündigt - nicht die Rede des Ich, die eine Sprache (das Ganze) zur Verfügung stellt. Die einzelnen Worte, die

12. — Park. Zeitschrift für neue Literatur, 4/1977, S. 27.

13. - Friedrich Hölderlin, „Andenken“, in: Ders., gesänge II, hrsg. von D.E. Sattler, Frankfurt a.M., Stroemfeld, 2000, S. 805.

14. - Martin Heidegger, „Wozu Dichter?“, in: Ders., Holzwege, Frankfurt a.M., Klostermann, 1977, S. 364. 
zur Hand gegeben werden, fügen sich im engen, vertikalen Raum zu einer Mitteilung zusammen, die besagt, dass Sprache eben nicht durch einen Willensakt, sondern erst dadurch bewohnbar wird, dass man ihre Bestandteile mit und in sich trägt, langsam und behutsam sich mit ihnen vertraut macht. Hermetisch ist an dieser Lehre vor allem, dass sie sich insgeheim selbst kontert, was man aber nur merkt, wenn man ihr Wort lange genug auf sich wirken lässt. Im Begriff der Dauer ist die Information enthalten, dass dieser Vorgang kein Ende nimmt.

Von diesem frühen Punkt an halten sich Muellers Gedichte stets im Spannungsraum zweier Pole auf: das Anbieten einer gemeinsamen Sprache einerseits und die kritische Reflexion über diese Geste, deren Voraussetzungen und Implikationen andererseits. Diese doppelte Bewegung einer kontinuierlichen Annäherung und Abstandnahme geht auf einen spezifischen biographischen Hintergrund zurück, der mit einer ebenfalls spezifischen, frühen Erfahrung mit der Sprache und ihrer Gebrochenheit einher geht. Mueller wurde in einer Familie von Einwanderern aus Mähren geboren, deren Deutsch sich stark von dem in Franken und in der Kurpfalz, wo er aufwuchs, gesprochenen, unterschied. Das Kind lernte nicht nur Nuancen innerhalb derselben Sprache wahrzunehmen, sondern bekam auch den sozialen Druck zu spüren, der die Anpassung entweder an die sprachliche Mehrheit oder aber vom Dialekt weg zum Hochdeutschen einfordert. Entscheidend war die Enthüllung der eigenen jüdischen Identität von Seiten der Großmutter mütterlicherseits, die sie der Tochter verborgen hatte. Diese wiederum hatte vor dem Krieg einen Mann geheiratet, der dann der SS beitrat und 1945 in sowjetische Gefangenschaft gelangte. Muellers leiblicher Vater floh kurz nach der Geburt des Sohns 1949 als Kommunist aus der Bundesrepublik nach Venezuela. Der Kriegsgefangene kehrte zurück, adoptierte das Kind und wurde zu seinem größten Peiniger, wie manche der härtesten Passagen in Muellers Gedichten bezeugen: „Ich erschlag dich / wenn du schreist / sagt mein Vater"15.

15. - Rainer René Mueller, Augen, Büdingen-Düdelsheim, pawel pan presse, 1983, S. 6, V. 16-18. In der Schreibung des vom Stiefvater geerbten Namens („Mueller“ statt „Müller") kommt subtil die Abgrenzung von diesem zum Ausdruck. Der Gedichtband Chasse de neige / Schneejagd (Stuttgart, Edizione Scampi, 1983) ist unter dem Namen Ellis Eliescher erschienen, unter Verwendung des Mädchennamens der Großmutter. Diese biographischen Informationen wurden mir im Laufe von zahlreichen Gesprächen mit dem Autor mitgeteilt. Ich danke Claudia Kramatschek dafür, dass sie mir das Typoskript eines langen Interviews zur Verfügung gestellt hat, das sie 2018 mit Rainer René Mueller geführt hat und in dem ebenfalls zahlreiche biographische und poetologische Informationen enthalten sind. Dazu auch: Stefan Ripplinger, ,Jäger im Schnee. Der jüdische Dichter Rainer René Mueller spiegelt Gewalt und Verletzung in der Sprache“", Neues Deutschland, 17. September 2016. Vgl. auch das frühe (1977?) unveröffentlichte Gedicht „Auf das Nähzeug meiner Mutter“: „Zuckersackstoff / aus Saaz / Lagergewebe / über die Jahre hin / ein Nadelkissen // ein Leichentuch / hat alle 
Das Hölderlin'sche Thema der väterlichen Herkunft, mit dem das „Adler"-Fragment beginnt ${ }^{16}$, nimmt in Muellers Leben einen unverkennbar traumatischen Charakter an, da sich darin die Gewaltsamkeit, die Brüche und unversöhnlichen Widersprüche der deutschen Kriegsund Nachkriegszeit in konzentrierter Form vorfinden. In einem evangelischen Umfeld aufgewachsen, suchte Mueller als junger Mann nach einer anderen sozialen und religiösen Zugehörigkeit und fand, nach einer Annäherung an den Katholizismus, erst nach und nach den Weg zu einem Judentum, zu dem er sich heute offen bekennt ${ }^{17}$. Es gibt keine Linearität in Muellers Leben, dem das Grenzgängerische von Geburt an und von der Geschichte mitgegeben wurde. Die rhetorische Figur, die diesem Leben entspricht, ist der Anakoluth, der am Ende des Gedichts „Chorus" einen prominenten Auftritt hat:

züchte Raben, dieses Anna
$\quad$-lied
Spanisches im Gewölbe
im Gewölbe
im bemalten Himmel
$\quad$-koluth, -koluth ${ }^{18}$

Evoziert wird der kindliche Blick auf die bürgerliche Familie während Francos Diktatur aus Carlos Sauras Film Cría cuervos (Züchte Raben, 1976). Der Name der jungen Protagonistin, Ana, ist im Gedicht auch der Name der Mutter Mariä, deren Lied über Umwege eine Verwandlung zur genannten rhetorischen Figur logischer Inkonsistenz durchmacht. Durch die Unterbrechung zeigt sich die eigentümliche Folgerichtigkeit eines auf dem Anakoluth beruhenden Liedes, da das griechische Adjektiv, das ,folgend“ bedeutet (akólouthos), vom negierenden Präfix gelöst, das Gedicht beendet und auf Kommendes vorbereitet. Der Anakoluth versinnbildlicht eine weitere Form jüdischer Identität nach der Shoah: aus Überlebensgründen verheimlicht, musste die vergessene, für eine Generation suspendierte jüdische Identität mühsam wieder entdeckt und rekonstruiert werden.

Das Befremdliche des Kompositums „LiedDeutsch“ ist Ausdruck dieses vielfach gebrochenen Lebenslaufs und der Haltung zur Sprache,

/ Stiche ertragen / und das Blut / vieler Fingerspitzen // ein ganz fester Stoff / auf dem ich / mir meine Tage / ankreide." Archiv Rainer Blum, Berlin.

16. - Vgl. F. Hölderlin, Einleitungsband, a.a.O., S. 43: „Mein Vater ist gewandert / (Gewandert) ist mein Vater auf dem Gotthard“.

17. - Die Frage der (unerwünschten) Taufe wird im Gedicht „Auferstehung“ ausdrücklich thematisiert: ,was war da / worin bin ich gewaschen // [...] beschlagt mich mit Wasser, mit gemahlenem Wasser / mit Staub“. R. R. Mueller, LiedDeutsch, a.a.O., S. 25.

18. — Ebd., S. 24. 
die daraus entstanden ist. Weder deutsches Lied noch Lied der Deutschen, ist der Begriff Ergebnis erstens einer Umkehrung, zweitens einer harten Fügung zweier Wörter, die einmal vielleicht guten Gewissens miteinander verbunden werden konnten und nun ohne jegliche Vermittlung aufeinanderprallen (müssen). Die Begegnung der Verschlusslaute erzeugt ein Stottern, das die Gewaltsamkeit des Aufpralls und die Unvereinbarkeit der beiden Wörter hervorhebt. Das Kompositum illustriert somit wortwörtlich Adornos Diktum, Gedichte nach Auschwitz zu schreiben, sei barbarisch. Statt es als Verbot zu begreifen, bringt Mueller in seinen Gedichten das Barbarische stets und unermüdlich in den Vordergrund.

War 1980 im Insel Verlag eine zweibändige Sammlung Deutsche Lieder mit Text und Partitur erschienen, so stellen das im selben Jahr veröffentlichte Gedicht „LiedDeutsch" ${ }^{19}$ und die im gleichnamigen Band erschienenen Texte, aber auch Muellers spätere Gedichtbände, deren entschiedenes Gegenprogramm dar ${ }^{20}$. Keiner der Texte entspricht der in der Einleitung zu Deutsche Lieder formulierten Definition, nach der ,[a]ls Lied [...] alle Gebilde gelten [sollen], die einen relativ kurzen, strophenmäßig geteilten Text übersichtlich durch Reimschema und Versfuß gliedern und deren lyrischer oder epischer Inhalt singend dargestellt wird."21. Obwohl sie fast durchgehend an diese Tradition anknüpfen, stellen sie die Möglichkeit in Frage, diese - zumal nach 1945 - ohne kritische Distanz, als wäre sie unbeschadet „durch die tausend Finsternisse totbringender Rede"22 hindurchgegangen, zu übernehmen. So heißt es ausdrücklich in den Schlussversen von „Vaterland, kornblumenblau“ aus dem dritten Band Augen (1983): „es ist ein Verhör in der Luft / ein Auge ist in der Luft // die Rüben

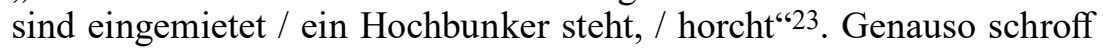
wie im Neologismus „LiedDeutsch“ stehen in diesem Gedicht Willy Schneiders Schlager „Kornblumenblau“ - ein Loblied auf das liebliche Leben am Rhein in Gesellschaft blauäugiger Frauen -, und „Gebrüll“

19. — Erstveröffentlichung in: Helmut Heißenbüttel, Bernd Jentzsch (Hrsg.), Hermannstraße 14. Halbjahresschrift für Literatur, 5/1980, S. 93.

20. - Der Band LiedDeutsch enthält 58 Gedichte, von denen manche in den Jahren davor bereits in Zeitschriften erschienen waren. Das titelgebende Gedicht „LiedDeutsch“" wurde als achtes ebenfalls im Band aufgenommen. Die früheren Gedichte lassen sich an der Kürze der Verse, an der Abwesenheit von Stropheneinteilung und Satzzeichen erkennen.

21. - Ernst Klusen (Hrsg.), Deutsche Lieder. Texte und Melodien, Frankfurt a.M., Insel Verlag, 1980, S. VI.

22. - Paul Celan, „Ansprache anläßlich der Entgegennahme des Literaturpreises der Freien Hansestadt Bremen", in: Ders., Der Meridian und andere Prosa, Frankfurt a.M., Suhrkamp, 1983, S. 38.

23. - R.R. Mueller, Augen, a.a.O., S. 30. Das Gedicht wurde in poèmes - poëtra, Solothurn, roughbooks, 2015, S. 30f., erneut abgedruckt. 
(V. 4), „Exilreden“ (V. 9), „die Göbbelsgosche“(V. 12) einander gegenüber. Das leichtfüßige Lied eines Sängers, der während und nach der Nazi-Zeit großen Erfolg genoss, läuft im Hintergrund weiter, während Totschlag und Gewalt genannt werden. Zurückhaltende Hinweise auf Paul Celans Gedicht „Du liegst“ erinnern an die Ermordung von Rosa Luxemburg und Karl Liebknecht ${ }^{24}$, während die darauffolgenden Verse weitaus expliziter sind:

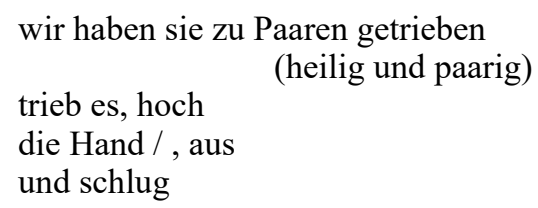

Der Kontrast ist demjenigen, der in Celans Gedicht zwischen Totschlag und Weihnachtsmarkt entsteht, ähnlich. In Muellers Text steht jedoch die Spannung innerhalb der lyrischen Tradition im Mittelpunkt. Die zurückhaltende Anwesenheit von „Du liegst“ ist einerseits eine Würdigung des poetischen Vorgängers; andererseits reflektiert sie die vermittelnde Funktion von Dichtung, jene Geschichten weiterzureichen, da ,im Tiergarten / die Stimme“(V. 10f.) - anders als etwa der genannte Reichspropagandaleiter - nicht ,zu tausenden Stimmen, die gafften“ (V. 13) schrie, und sich folglich die Frage der Zeugenschaft dringend stellt.

In „Vaterland, kornblumenblau“ ist es die spätestens seit Novalis in der deutschen Lyrik prominent gewordene Farbe Blau, „die Stimmen verbietet, Gebrüll“" (V. 4), als wäre die blauäugig metaphysisch aufgeladene Bedeutung dieser Farbe mit dafür verantwortlich (gewesen), dass gewisse Stimmen kein Gehör bekommen (haben). Die sich immer wieder von Neuem entfesselnde Gewalt wird zur innerpoetischen Angelegenheit. Wenn es nach der expliziten Nennung physischer Gewalt heißt: „mal sind diese Stimmen auch nah und ganz“ (V. 20), ist die Erleichterung ob der Nähe und Unversehrtheit nur vorübergehend, steht doch das Adjektiv „blau“ isoliert (und damit hervorgehoben) im darauffolgenden Vers, um die Stimmen durch seine Mehrdeutigkeit zu kennzeichnen („mal sind diese Stimmen auch nah und ganz / blau“). Sie sind blau, weil soeben eine Hand zum Schlag ausholte, und blau, weil sie, der Aufforderung Willy Schneiders nachkommend (,darum trinkt Rheinwein, Männer, seid schlau“), zu viel getrunken haben ${ }^{25}$.

24. — Der fünfte Vers, ,:staken, hüten: hecheln“ in Verbindung mit der Erwähnung des Tiergartens (V. 10) schafft ein feines poetisches Netz, das die „Äppelstaken“(V. 5) und den Landwehrkanal (V. 12) aus Celans ,Du liegst“ mit einbezieht (Paul Celan, Die Gedichte, hrsg. von Barbara Wiedemann, Frankfurt a.M., Suhrkamp, 2018, S. 485). Die Anwesenheit Celans ist in Augen besonders stark; das Gedicht „da in der Rue d'Ulm“ (S. 31) widmet sich ausdrücklich Celans Leben und Tod in Paris.

25. — „Vaterland, kornblumenblau“ greift hiermit Elemente aus ,gehst du...“, 
Wie die Andeutungen auf Volkslied und Gedicht wechseln sich die zum Schweigen gebrachten Stimmen mit den Stimmen ab, die sagen: „wir haben sie zu Paaren getrieben“(V. 15), oder: ,du kommst nicht vor" (V. 23), in einem alptraumartigen Rausch, der mit der oben erwähnten Vision eines wohl notwendigen ,Verhör[s] in der Luft“"26, vor allem aber mit dem fordernden Verb „horcht“ zum vorläufigen Schluss kommt. Das Horchen gilt den Unterschieden zwischen Stimme und Stimme, Stille und Gebrüll, die im Vaterlandssuff zu verschwimmen drohen. Die Bewegung von der Beschreibung der lieblichen deutschen Natur, die im Blau der Kornblume sinnbildlich Gestalt annimmt, zur vom Rheinwein verursachten heiteren Trunkenheit, vollzieht sich bereits in Schneiders Lied, in dem ,kornblumenblau“ sowohl für letztere als auch für den (deutschen) Himmel und die Augen der (deutschen) Frauen steht ${ }^{27}$. Mueller brauchte sie nur zu übernehmen und auf ihre ungeheure Spitze zu treiben, indem er sie mit anderen, die künstliche Heiterkeit verstörenden Bildern kollidieren ließ.

\section{Gesang, Lied, Schrei}

Zum lustvollen Singen eignet sich ein solches Lied nicht. Gleichwohl lässt sich an der Verteilung der Verse eine Kompositionstechnik beobachten, die sowohl Celans „Engführung“ als auch der Mehrstimmigkeit mittelalterlicher Musik verpflichtet ist und sie auf eigene Weise einsetzt. Diese Vorgehensweise, durch die verschiedene Stimmen im Gedicht erkennbar und mithilfe von Klammern und Umbrüchen voneinander unterscheidbar werden, hat Mueller seit LiedDeutsch entwickelt und verfeinert; im letzten Band geschriebes kommt sie vollends, durchgehend und mit großer Präzision zur Geltung 28 . Ähnliches gilt für die Zeichensetzung, da etwa Schrägstriche und Doppelpunkte in unterschiedlicher Entfernung vom vorangehenden oder folgenden Wort Pausen unterschiedlicher Länge markieren. Diese Trennungen bestimmen den rhythmischen Verlauf des Textes auf eine Weise, wie es in musikalischen Partituren

dem ersten Gedicht aus LiedDeutsch, a.a.O, S. 7, auf, in dem es heißt: „ein letzter und noch einer / ein Schlag geschrien / [...] glücksfähig Kinder seid / ihr es sei denn / ihr treibet ab / mit dem April dem ersten / geratet in Haumichblau“ (V. 9-10 und V. 18-22).

26. - Muellers „Verhör in der Luft“ könnte ein Widerhall von Celans „Grab in den Lüften“ aus der „Todesfuge“ sein.

27. - Die dritte Strophe von Schneiders Lied schildert eine deutsche Idylle: „Es gibt kein Plätzchen auf Erden, / Wo sich's so herrlich und fein / Lebt wie am Rhein, wo die Reben / Blühen im Sonnenschein. / Reich an Farben, so bunt und so prächtig / Erstrahlt Wald und Flur, / Von den Farben am Rhein / Eine allein tritt ganz besonders hervor." Die hervortretende Farbe ist natürlich das Blau.

28. - Dort enthalten acht Gedichte bereits im Titel oder Untertitel explizite musikalische Hinweise. 
der Fall ist ${ }^{29}$. Je mehr die Gedichte bewusst die Reflexion über das (deutsche) Lied aufnehmen, desto mehr verlieren sie ihre anfängliche Linearität, desto häufiger werden die Einschübe, desto gebrochener sind scheinbar die Texte, die aber immer aufs Genaueste kompositorisch durchdacht sind. Während Celan sich bereits in der MeridianRede deutlich vom Lyrischen distanzierte ${ }^{30}$, hat sich Mueller vorsätzlich der systematischen Dekonstruktion des Liedes im Gedicht und also dessen lyrischer Dimension zugewandt. Von diesem Vorgang trägt dieser „deutsch[e] Gesang“31 - der von der Überlebensschuld mit verursacht wurde 32 - eindeutig Spuren.

Die fünfte, zweizeilige Strophe von „Lirum, larum“ illustriert diese Vorgehensweise mit einer Abfolge, die Klangähnlichkeiten ausschöpft, um auf Abgründe in der Geschichte der deutschen Sprache und Poesie, in der deutschen Geschichte überhaupt zu zeigen. Die Reihe beginnt mit Julius Streichers 1923 in Nürnberg gegründeter antisemitischer Zeitschrift Der Stürmer, deren aggressiver Name durch Verschiebung der Anfangsbuchstaben zu den von ihr mitveranlassten Zerstörungen der „Stunde Null“ führt: „,das Stürmerlied, das Türmerlied / das Trümmerlied, ach Walther" (V. 17-18)33. Die klagende, vertrauensvolle Anrufung des zuvor im Gedicht indirekt bereits genannten Walther von der Vogelweide 34 unterbricht jäh die Steigerung, als müsste gerade der Gründungsvater deutscher Poesie das Entsetzen über ihr Schicksal teilen. Diese Verse sind eingebettet in eine schwindelerregende Verschränkung von Verweisen auf für das (vermeintlich) Deutsche maßgebliche Künstler (Albrecht Dürer, Walther von der Vogelweide, Tilman Riemenschneider, Johann Sebastian Bach), Kunstformen (Dichtung, hohe Musik, bildende Kunst, Volkslied) und Epochen - vom Mittelalter bis in die jüngste Vergangenheit hinein. Diese Verschränkung wirkt wie ein Kurzschluss, wirft aber zugleich

29. - In dieser Hinsicht erweist sich Muellers Lyrik als die systematische Umsetzung folgender Bemerkung Adornos: „In keinem ihrer Elemente ist die Sprache so musikähnlich wie in den Satzzeichen.“ Theodor W. Adorno, „Satzzeichen“, in: Ders., Noten zur Literatur, a.a.O., S. 106f.

30. - Vgl. Paul Celan, Der Meridian. Endfassung - Entwürfe-Materialien, hrsg. von Bernhard Böschenstein und Heino Schmull, Frankfurt a.M., Suhrkamp, 1999, S. 151.

31. - Friedrich Hölderlin, „Patmos“, in: Ders., gesänge I, hrsg. von D.E. Sattler, Frankfurt a.M., Stroemfeld, 2000, S. 445.

32. - Vgl. das Gedicht „Wege, Wege“: „Geräusche / das Wort Überlebensschuld // etwas unterbricht uns, verursacht diesen Gesang“". R.R. Mueller, LiedDeutsch, a.a.O., S. 26, V. 1-3.

33. - Ebd., S. 10.

34. - In der zweiten Strophe werden „die geschränkten Beine / des Vogel, -Sängers“ genannt, die den berühmten Anfang von Walthers Gedicht zitieren: „Ich saz ûf eime steine / und dahte bein mit beine, / dar ûf satzt ich den ellenbogen“. 
ein grelles Licht auf Überlappungen, die zunächst semantisch sind, gleichwohl dringende Fragen stellen: in welchem Zusammenhang steht Walthers politische Dichtung (,Reichston“, V. 15) mit dem Ton des Dritten Reichs, dem antisemitischen Ton vom Stürmer? Auf die Gewalt der Nazi-Zeit deuten vor allem die Schlussverse hin (,darum, dass einer ein Vieh, ein gehäutetes Vieh / wird, ein Stück, ein Hakenstück“), die auf einen Besuch des Autors in der Justizanstalt Plötzensee zurückgehen. Dort wurden ab Ende 1942 Hinrichtungen durch Hängen an Stahlträgern und Eisenhaken vollzogen. Wie lässt sich der Kontrast zwischen diesen Ereignissen und Walthers berühmtem Liebesgedicht „Unter der Linden“, aus dem in „Lirum, larum“ der Refrain „tandaradei" zitiert wird, ertragen? Was - so die gnadenlose Frage in der sechsten Strophe - heißt Liebe, vor diesem Hintergrund? Walthers Liebe(slied) wird zum Stammeln: „was heißt hier Liebe // -belei, -lei / larum, larum :“(V. 20-22).

Im späteren Gedicht „Gehäuse“ wird mit den Versen „Lied : frei / stammeln" 35 die Befreiung des Liedes im Stammeln ausdrücklich festgelegt. Die Verwandlung des Gesangs zum gestammelten Abgesang erfährt eine weitere Radikalisierung in zwei Gedichten aus Augen, die das Verhältnis von Gesang, Schreiben und Schrei zum programmatischen Mittelpunkt von Muellers Lyrik machen. In Anlehnung an die konkrete Poesie und vor allem nach dem Beispiel von Ernst Jandls „Wien: Heldenplatz“ und „schtzngrmm“ schöpft Mueller die lautlichen Möglichkeiten der Sprache aus, um zu zeigen, dass im Schreiben der Schrei und in der französischen écriture der cri immer schon bereits enthalten $\operatorname{sind}^{36}$.

In der „Vogel-Sequenza“ wird der später wiederholte Anfangsvers, der zunächst syntaktische Kohärenz verspricht, schrittweise zur Onomatopöie verwandelt, die in reduzierter Gestalt den Inhalt des Verses illustriert:

Wie ein Vogel über die Ebene schreit schreit ein Vogel, wie

er, er

schrier, schrier ${ }^{37}$

35. - R.R. Mueller, Augen, a.a.O., S. 8, V. 5f. Das Gedicht beginnt mit einem Hinweis auf Hölderlin: „,die Vokalreihe / ,Jaunerloh gebi... // Holdrio, Holder- / lein“. 36. - Es handelt sich um die Gedichte „Vogel-Sequenza“ und „Munch-Stück“. Aus Platzgründen werde ich nur auf ersteres eingehen, obwohl das zweite die unheimliche Verbindung von Religion, väterlicher und nationalsozialistischer Gewalt und Sprache auf noch radikalere Weise zur Schau stellt.

37. - Ebd., S. 6, V. 1-4. Wie es auch bei Jandls Lautgedichten der Fall ist, kommen diese Gedichte in Muellers eigener Lektüre mit besonderer Kraft zum Leben. 
Die Erwartung auf die Fortsetzung des Vergleichs wird nur auf syntaktisch-logischer Ebene enttäuscht, da der Leser stattdessen den Schrei unmittelbar zu Gehör bekommt. Der Vogel ergreift das Wort und widersteht somit der objektivierenden Beschreibung des ersten Verses. Es ist der Schrei dieses Vogels - ein Erbe von Hölderlins Adler, wie auch einer von Sauras undankbaren Raben ${ }^{38}$-, der die sich souverän gebende Rede stört und dem inartikulierten Ausruf einen würdevollen Platz im Gedicht einräumt. Er erweist sich im Verlauf des Gedichts als Schrei der Klage und der Anklage, die Reaktion auf die bereits erwähnte väterliche Gewalt:

\section{Ich schrei - -be, -be \\ Bilder}

Ich erschlag dich wenn du schreist sagt mein Vater:

Schreien, Stammeln, Schreiben und Beschreiben sind zum Akt des Widerstands konzentriert. Der Dreizeiler illustriert bündig die Einschätzung des russischen Philosophen Lev Šestov, dass Klage und Schrei eine eigene, von der logisch stringenten Artikulation sich unterscheidende „Dimension des Denkens“ erschließen ${ }^{39}$. Das Gedicht nimmt allerdings ein unerwartet bitter-ironisches Ende, wenn es zur anfänglichen Formulierung zurückkehrt und sie mit dem Volkslied „Kommt ein Vogel geflogen“ in Verbindung setzt (,Schreit ein Vogel / wie er : Liebstengruß“،, V. 22f.). Dort hat der Vogel die Aufgabe, der Geliebten „ein'n Gruß [...], einen Kuß“ zu bringen, weil der Sprechende selbst ,ihn nicht begleiten [kann], [...] hier bleiben muß.“40. Die Botschaft des Vogels - des Sängers, des Dichters - ist, so die Botschaft der „Vogel-Sequenza“, keine der Zuneigung und Sehnsucht mehr, sondern eine des verzweifelten Schmerzes und Entsetzens. Ob diese wie jene den Adressaten erreichen werden, muss offenbleiben, wie es das zweite Gedicht aus LiedDeutsch, ebenfalls nach einem Volkslied benannt (,der Mai ist 'kommen“), unumwunden festhält:

38. - Der Titel von Sauras Film ist das erste Glied folgenden spanischen Sprichworts: „Züchte Raben, und sie werden dir die Augen ausreißen.“

39. - Vgl. Leo Schestow, „Die Nacht zu Gethsemane“, in: Ders., Auf Hiobs Waage. Über die Quellen der ewigen Wahrheiten, Berlin, Lambert Schneider, 1929, S. 440. Über Šestovs Verständnis des epistemologischen Gehalts der Klage habe ich ausführlich in meinem im Winter erscheinenden Buch Opak. Schatten der Erkenntnis in Paul Celans ,,Meridian" und im Gedicht ,Schwanengefahr", Göttingen, Wallstein, 2019, geschrieben.

40. - E. Klusen (Hrsg.), Deutsche Lieder, a.a.O., S. 329. 
keiner fragt, ob's schwer war

was schlug

ich brüllte shalom hin

dort hin und hier

$[\ldots]$

wir kennen den nicht

der uns zuhört

wir gehören ihm nur

in seinem $\mathrm{Ohr}$

welcher

wie Kinder schreien, diese Genauigkeit

mitten im Ruf, -

$$
\mathrm{da}, 41
$$

„Lirum, larum“ nennt gleich im Titel ein berühmtes Volkslied, das sich ebenfalls in der Sammlung Des Knaben Wunderhorn findet und mit dem fröhlichen Vers endet: „Ey was ein lustig Leben!“42 Es sind die für die rhythmische Gestalt des Liedes grundlegenden, rein lautlichen und semantisch leeren Wörter „tandaradei“ und „lirum larum“, die in Muellers Gedicht jene Assoziationskette tragen, die schließlich zum Binnenreim „larum ... darum“ und damit zu einer syntaktisch wieder (fast) vollständigen Aussage führt. Auf welches „,warum“ aber könnte das brutale „darum“ der letzten Strophe Antwort geben? Es handelt sich vielleicht um ein offenes, verzweifeltes ,warum?“, das einem aus Ohnmacht angesichts der in den wenigen Versen zusammengeschnürten deutschen Geschichte entfährt. ,deutsch, das ist auch“ - all das.

Das Gebot aus „Vaterland, kornblumenblau“, zu horchen, gilt nicht nur den singenden, brüllenden, oder aber den verstummten, zurückhaltenden Stimmen der Vergangenheit, sondern auch dem Gedicht selbst in seinen teils überlauten, teils leisen Übergängen, wenn beispielsweise auf das ,sie sagen: / du kommst nicht vor“, die Antwort lautet: ,(ich nicht)“(V. 23f.). „Ich [sage das] nicht" und ,ich [bin] nicht" (weil andere mir die Existenz absprechen), Widerstand und Untergang kommen sich hier unheimlich nahe. Unter Leichtigkeit versprechenden Titeln wie „LiedDeutsch“, „Lirum, larum“ und „Vaterland, kornblumenblau“ macht sich eine Bedrücktheit angesichts der Geschichte, insbesondere

41. — R.R. Mueller, LiedDeutsch, a.a.O., S. 8f., V. 7-10; 20-28.

42. - „Lirum larum Löffelstiel, / Alte Weiber essen viel, / Junge müssen fasten, / Brot liegt im Kasten, / Messer liegt daneben, Ey was ein lustig Leben!" Achim von Arnim, Clemens Brentano (Hrsg.), Des Knaben Wunderhorn. Alte deutsche Lieder, hrsg. von Heinz Rölleke, Stuttgart u.a., Kohlhammer, 1979, Bd. 3, S. 274. 
der Geschichte des Deutschen und ihrer Gegenwart breit, die Muellers Werk bis heute nicht verlassen hat und es zu einer steten Befragung seiner Hinter- und Untergründe anhält, dem Spruch aus dem Gedicht „November, November" treu: ,ich werd' nicht ruhig / in den Wörtern: Farben // das war die Losung "43.

\section{Struthof}

Im Gedicht „La Hutte, abends“44 tritt das Anfangsmotto Hölderlins im letzten Vers mit leichten, bedeutenden Abweichungen auf. Der im Eröffnungsgedicht von LiedDeutsch angekündigte Gang 45 führt in die Vogesen. Mit La Hutte ist der konkrete Ort genannt, an dem das Gedicht entstand - eine einfache, oberhalb von Schirmeck gelegene Jagdhütte, in die sich Mueller in der Zeit regelmäßig zurückzog. Die umgebende Natur findet gleich im ersten Vers Erwähnung: „der Wald steht" ${ }^{\text {"46. Es }}$ folgt eine Landschaftsbeschreibung, die den Gang durch den Wald abzeichnet, bei dem der Wanderer auf Überraschendes stößt: ,aus Wurzelholz ein gebleichtes Stück / aleph hinter Steinen“ (V. 5-6). Es ist

43. - R.R. Mueller, Augen, a.a.O., S. 42, V. 14-16. Diese Verse markieren eine Umorientierung auch im biographischen Sinne, da Mueller 1982 Leiter der städtischen Galerie in Schwäbisch Hall wurde und sich zunehmend der Gegenwartskunst widmete. Die intensive Auseinandersetzung mit der bildenden Kunst (vor allem mit der zeitgenössischen) und die stete Zusammenarbeit mit Künstlern prägten im Laufe der 1980er Jahre zunehmend auch die poetische Produktion Muellers. Dieser Austausch ist u.a. an der Gestalt der Gedichtbände zu beobachten. Augen enthält Radierungen von Sascha Juritz, der Band Rückzug ins Helle (Edizione Scampi, Verlag Mario Haith, Stuttgart, 1985) geht auf die Zusammenarbeit mit dem Maler Max Neumann während eines gemeinsamen Aufenthalts in Berlin zurück und enthält zahlreiche Abbildungen von Neumanns Gemälden.

44. - R.R. Mueller, LiedDeutsch, a.a.O., S. 27. Dort ist es das 19. Gedicht; es besteht aus 28 Versen. Neu abgedruckt in: Ders., poèmes - poëtra, a.a.O., S. 14f.

45. - Das erste Gedicht von LiedDeutsch trägt den Titel „Gehst du...“ und antwortet unmittelbar auf Hölderlins „Der Adler“. Dort heißt es: „Der Urahn aber / ist geflogen über der See.“ (Hölderlin, gesänge I, a.a.O., S. 449.) Mueller erwidert entschlossen: „nein, / nur nicht fliegen / gehn sollst du / einen Schritt machen“ (V. 14-17). Auch hier treffen hohe und niedere Kultur, Volkslied und „deutscher Gesang“" aufeinander, da am Ende des Gedichts das Lied „Maykäfer, flieg!“ erwähnt wird, das wiederum auf Kriegsgeschehen und auf die Involvierung der Eltern darin Bezug nimmt: „Maykäfer, flieg! / Der Vater ist im Krieg / Die Mutter ist im Pulverland / Und Pulverland ist abgebrannt.“ A. von Arnim, C. Brentano (Hrsg.), Des Knaben Wunderhorn, a.a.O., Bd. 1, S. 221. Hölderlins Adler verwandelt sich in einen Maikäfer, Hölderlins wandernder Vater verwandelt sich in den im Krieg dienenden Vater aus dem Volkslied.

46. - Vgl. eine E-Mail von Mueller an die Verf. vom 7. März 2019. Das Gedicht bezieht sich u.a. auch auf das berühmte „Abendlied“ von Matthias Claudius. Von der dortigen nächtlichen Idylle setzt sich die Landschaftsbeschreibung in „La Hutte, abends“" scharf ab. Mueller selbst hat das Gedicht als ,ein einziges gegenlied gegen die Idylle des M Claudius Abendlied" beschrieben (E-Mail an die Verf., 7. März 2019). 
eine „Fundsache“, die „unten [...] im Steinbruch / im rötlichen Basalt“ liegt. Der erste Buchstabe des hebräischen Alphabets bleibt zunächst nur eine Andeutung. Diese Andeutung verliert ihre scheinbare Willkür, sobald man in der vierten der sechs Strophen angelangt ist:

Es brennt uns hinter die Kämme

$$
\begin{aligned}
& \text { : das Mal, das Denkmal } \\
& \text { Le Struthof, Holz und Zimmermann : }
\end{aligned}
$$

Dort wird der Name des elsässischen Konzentrationslagers Natzweiler-Struthof genannt, das vom 21. Mai 1941 bis September 1944 in Betrieb und von Muellers Unterkunft sichtbar war ${ }^{47}$. In der Nacht vom 13. auf den 14. Mai 1976 setzten Neo-Nazis das Museum, das auf dem Gelände errichtet worden war, in Brand. Es wurde am 29. Mai 1980 wieder eröffnet ${ }^{48}$. Das „Denkmal“ ist das 41 Meter hohe Mémorial national de la Déportation, das 1960 dort eingeweiht wurde. Das Monument in der Gestalt einer Flamme symbolisiert das nie auszulöschende Gedenken an die Opfer. Das „es brennt“ dürfte sich sowohl darauf als auch auf das Krematorium des KZs beziehen. Die Konkretheit des Namens entledigt die natürliche Szenerie ihrer Unschuld, denn im Steinbruch, der in der zweiten Strophe erwähnt und in dem das ,aleph“" gefunden wird, mussten die KZ-Insassen schwerste Zwangsarbeit leisten. Die ,geschälte[n] Stämme hangabwärts“, die in der dritten Strophe genannt werden, erhalten ebenso rückwirkend ihren spezifischen Sinn. Die Insassen mussten in Zweigstellen von Rüstungsfirmen arbeiten, die zum Schutz vor Luftangriffen im Inneren des umgebenden Gebirges aufgerichtet wurden. Ein Schwarz-WeißBild zeigt den Eingang zu einem solchen Werk, über dem ,geschälte Stämme hangabwärts" (V. 12) querliegen.

Es gibt im Gedicht vier weitere stark eingerückte, durch Doppelpunkte eingerahmte Verse. Sie fungieren als kommentierende Gegenstimme innerhalb der Strophe, in die sie jeweils eingebettet sind. Unter ihnen findet sich Hölderlins Vers abgewandelt wieder. Im Kontext der Beschreibung vom Steinbruch heißt es: „,: und weidet mich :“(V. 11). Dann in der letzten Strophe:

es palavert wo und unten zu Strasbourg

: auf der Schanz' :

47. - Vgl. Robert Steegmann, Das KZ Natzweiler-Struthof und seine Außenkommandos an Rhein und Neckar, 1941-1945, Berlin, Metropol-Verlag, 2010.

48. - Struthof wird auch in den Gedichten „Drei Stücke im Volkston“ (poèmes - poëtra, a.a.O., S. 92) und „Arkonaplatz, Berlin, und anderswo geseijt“ (geschriebes, a.a.O., S. 13) erwähnt. Mueller hat lange Zeit zwischen Straßburg und dem Dorf Harbouey nahe den Vogesen gelebt. 
da trommeln sie

die neuen Lieder. Sie pfeifen

sie weiden aus :

die Quadrille, sie stellen Wände auf

der Psalter steht im Korn.

Es ist zu Weide gut.

Die Aufeinanderfolge dieser um das Verb „weiden“ kreisenden Verse zeigt beispielhaft das jäh verdichtende Verfahren, das für Muellers Lyrik kennzeichnend ist und für gewöhnlich auf Abgründe und unversöhnliche Widersprüche hindeutet. Vers 11 zitiert den 23. Psalm in der Übersetzung Luthers: „,Der Herr ist mein Hirte; mir wird nichts mangeln. / Er weidet mich auf einer grünen Aue und führet mich zum frischen Wasser." ${ }^{\text {"49 }}$ Der Kontrast zum Kontext, in den das zuversichtliche biblische Zitat eingelassen ist, könnte stärker und erschreckender nicht sein. Fast gnadenlos zeigt er auf die Vergeblichkeit und Sinnlosigkeit des Gebets zu einem Zeitpunkt, da Menschen wortwörtlich und keineswegs im übertragenen Sinne als Vieh behandelt wurden und das „Weiden“ ein Euphemismus für Zwangsarbeit wird.

Die vierte „Gegenstimme“ ist ebenfalls ein Zitat, aus dem Volkslied „Zu Straßburg auf der Schanz". Darin erzählt ein Deserteur seine durch die Nostalgie veranlasste Flucht ${ }^{50}$, die Festnahme und anstehende Hinrichtung. Das Lied hebt das letzte Wort des Erzählers vor dem Tod für die Zukunft auf. Im August 1943 wurden die Leichen von 86 jüdischen Häftlingen, die in der Gaskammer von Struthof ermordet worden waren, zum Anatomischen Institut der Reichsuniversität Straßburg gebracht, damit der Anatom August Hirt aus ihnen eine Skelettsammlung im Rahmen des rassistischen Ahnenerbe-Projekts anlegen konnte - ihre Identität wurde erst 2003 bekannt gemacht ${ }^{51}$. Das Lied endet mit dem Wort: „Das klag' ich an“, doch die Anklage bezieht sich nicht mehr nur auf die Hinrichtung des unbekannten Deserteurs, sondern durch das Gedicht hindurch auch auf die Opfer nationalsozialistischer Gewalt. In dieser Hinsicht ist „La Hutte, abends“, wie viele andere Gedichte Muellers, als lautstarkes J'accuse zu verstehen, das Fakten, Orte und Daten in Erinnerung ruft und den Leser zwingt, sich auf ihre Spur zu begeben, diese Wege zu gehen.

49. - Die Bibel, nach der Übersetzung Martin Luthers, Stuttgart, Deutsche Bibelgesellschaft, 1999, S. 562.

50. - „Der Hirtenbub ist doch schuld daran, / Das Alphorn hat mir solches angetan“. A. von Arnim, C. Brentano (Hrsg.), Des Knaben Wunderhorn, a.a.O., Bd., 1, S. 136f. Auch: E. Klusen (Hrsg.), Deutsche Lieder, Bd. 2, a.a.O., S. 461f.

51. - Vgl. Hans-Joachim Lang, Die Namen der Nummern. Wie es gelang, die 86 Opfer eines NS-Verbrechens zu identifizieren, Hamburg, Hoffmann und Campe, 2004. 
Der letzte Einwurf (,,: sie weiden aus :") ist eine grausame Variation zum Verb ,weiden“, mit der Bedeutung ,einem geschlachteten, erlegten Tier die Eingeweide entnehmen" 52 . Die Leichen der 86 Häftlinge wurden zwar nicht mehr für Hirschs pseudo-wissenschaftliche Zwecke präpariert; dass es allerdings die Absicht war, sie auszuweiden, steht außer Zweifel. Liest man die Aussage zusammen mit dem darauffolgenden Vers, dann heißt es von den ,,neuen Lieder[n]“, die ,auf der Schanz"“ getrommelt werden, auch, dass sie ausgeweidet, gleichsam mit Gewalt ausgehöhlt werden. Wer aber sind ,sie“? Wer ist das Subjekt dieser musikalischen Handlungen (,sie pfeifen / die Quadrille“), um welche „neuen Lieder“ handelt es sich? Sind es solche, die - anders als manche alten Lieder - die Pflicht zur Anklage verweigern? Die letzten zwei Verse bringen die Anklage schließlich durch die Ausnutzung einer weiteren Doppeldeutigkeit zu einem stockenden Höhepunkt. Wie am Anfang der Wald, so steht am Ende der Psalter ,im Korn“. „Korn“ bezeichnet aber, außer dem Getreide, auch jenen kleinen Teil an der Mündung einer Handwaffe, die als Orientierung für das genaue Zielen dient. Daher stammt die Redewendung ,jemanden, etwas aufs Korn nehmen“ im Sinne von „etwas angreifen, heftig kritisieren“53. Das Gedicht kommentiert sich selbst, denn was war der Einschub ,und weidet mich" anderes als eine solche, durch die Positionierung des Zitats bewirkte Polemik gegen das Versprechen des Psalms („ob ich schon wanderte im finstern Tal, fürchte ich kein Unglück; denn du bist bei mir")? Weil die Lieder ausgeweidet sind, weil Struthof war, steht auch, steht selbst der Psalter unter Anklage. Die Feststellung, ausgerechnet dieses Korn sei „zur Weide gut“, ist ein weiterer Ausdruck bitterer Ironie. Gerade weil dieses nach Gewalt schmeckende Korn seinem Anschein zum Trotz nicht zur Weide gut sein kann, soll noch darf, bestätigt sich Hölderlins paradoxe Intuition, dass man sich lieber am Spärlichen, Kargen (am Fels) ernähren soll, das die Spuren der Vergangenheit aufbewahrt.

\section{Steinbruch, Geschriebes, Kassiber}

Im Gedicht „... Schöntrauer, winters“ aus geschriebes wird die gleiche Stelle aus dem 23. Psalm nach 35 Jahren stockend erneut erwähnt:

Todt'n-

au; -berg : ( aus- ) weidet /

mich

„,auf grüner Aue“ : Psalmen-

52. - Duden, s.v. ausweiden.

53. - Duden, s.v. Korn. 
Weinen

$$
\text { ,estre“ —54 }
$$

Das Zitat ist zugleich ein Selbstzitat, eingelassen in einen Kontext philosophischer Reflexion und Kritik (an Heidegger). Seine Anwesenheit erklärt sich zum einen durch die „Aue“, die sich im Ortsnamen Todtnauberg versteckt und in Vers 20 durch Trennung isoliert wird; zum anderen ist sie durch die Frage gerechtfertigt, mit der das Gedicht beginnt und die sowohl an das Hölderlin-Motto als auch an die ontologische Problematik des frühen Gedichts „Vom Hermetischen“ erinnert:

... der Schein trügt, der Fragen ...

Stein sein ? - sein sein, ach Stein. Geode, Sein

$$
\text { ,estre“ - }
$$

Motive aus mehreren Jahrzehnten werden in diesem Text erneut hinterfragt und führen schließlich zum Bekenntnis des letzten Verses: „Steinchen : Sein“.

Die programmatische Hinwendung zum Stein, für die Mueller mit der Übernahme des Worts ,der Fels ist zu Weide gut“ eingetreten war und die er in den Titeln der letzten zwei Bände poèmes - poëtra (2015) und geschriebes. selbst mit stein (2018) ausdrücklich hervorhebt, hat wie die Frage: was ist deutsch? - eine lange und reichhaltige poetische Tradition. In Osip Mandel'štam, der seinem ersten Gedichtband bekanntlich den Titel Stein (Kamen', 1913) verlieh, und Paul Celan hat sie zwei berühmte Vertreter im 20. Jahrhundert. Celans Verse aus „Engführung“ könnten denjenigen aus Hölderlins „Adler“ zur Seite gestellt werden: „Es blieb / Zeit, blieb, / es beim Stein zu versuchen er / war gastlich, er / fiel nicht ins Wort." 55 .

In einem unveröffentlichten Typoskript, das auf den 23. November 1978 „im Zug nach Berlin“ datiert ist, lässt sich eine Stellungnahme von Mueller zu diesem Thema nachlesen, die es erlaubt, einen Bogen zum Titel geschriebes. selbst mit stein zu schlagen und ihn auszulegen. Sie lautet: „Eine persönliche Bescheidenheit entwickeln, die opponiert, ohne mehr sein zu wollen, als Selbst. Eine Person wie ein Steinbruch. ${ }^{\text {"56. }}$. Dieser Grundsatz hat in Muellers Leben und Werk bis heute nicht an Gültigkeit verloren. Der Steinbruch, unter anderem bekannt als Ort von Zwangsarbeit sowie von K.s Hinrichtung in der Schlussszene von Kafkas Process ${ }^{57}$, wird hier zur bewusst angenommenen Haltung

54. - R.R. Mueller, geschriebes, a.a.O., S. 49, V. 19-24.

55. - P. Celan, Die Gedichte, a.a.O., S. 119.

56. - Typoskript, 23. November 1978, Archiv Rainer Blum, Berlin.

57. - Franz Kafka, Der Process. Ende, hrsg. von Roland Reuß und Peter Staengle, Frankfurt a.M., Stroemfeld, 1997, S. 18. 
eines Menschen, der unbeweglich und widerständig in sich ruht, aus dem aber durch Sprengung und Brechung (d.h. Verletzung) nach und nach Material abgetragen wird, das etwa für den Bau anderer Gebilde verwendet wird. In diesem Sinne wäre alles, was wir Menschen aus einer solchen Haltung heraus von uns in die Welt geben, und insbesondere ein Gedicht, ein solcher im Umlauf stehender Abtrag, von dem wir nicht genau vorhersehen können, was aus ihm wird. Daraus lässt sich weiterhin u.a. zweierlei entnehmen: erstens bestehen wir unsererseits schichtweise aus dem Gestein, das von anderen abgetragen wurde, wobei es aufgrund seiner Widerständigkeit als solches erkennbar bleibt (Mueller hat es so formuliert: „im Zitat find'st dich, immer : / du bist eins; / man nennt es Glück ..."58); zweitens liegt das Gestein in unterschiedlicher Dimension in der Welt vor (Steine oder Steinchen), auf dass der Sammler es auflese. Der Entstehungsprozess von Muellers Gedichten, deren heterogene Bestandteile er zunächst auf kleinen Zetteln notiert ${ }^{59}$, bis er daraus langsam einen einheitlichen Text komponiert, der die Spuren der Brüche nicht kaschiert, illustriert diesen Vorgang. Welche Steinchen in welche Hände geraten, bleibt dem Zufall überlassen, wie die Hinweise auf und das Zitat aus Mallarmés Un Coup de dés in ,... Schöntrauer, winters“ bezeugen ${ }^{60}$.

Wie man mit den gesammelten Steinen und Steinchen umgeht und wie man sie als Bausteine in sich aufnimmt, legt eine Notiz aus demselben Konvolut nahe, die auf den 13. September 1978 datiert ist:

So bleibt es also dabei: sich Kassiber von außen durch die Rippen schieben, schieben lassen und darauf hoffen, wirklich hoffen, und sei es nur für einen kurzen Augenblick der Zeit, die uns zur Verfügung steht, daß einer ein Stück mitgeht ${ }^{61}$.

Der gaunersprachliche Begriff Kassiber kommt vom Jiddischen kessovim, das seinerseits vom Hebräischen ketavim (Geschriebenes) hergeleitet ist. Es handelt sich um ein „,heimliches Schreiben oder [eine] unerlaubte schriftliche Mitteilung eines Häftlings an einen anderen oder an Außenstehende“62. Einen Tag später notiert Mueller: „Die

58. — R.R. Mueller, ,... man nennt es Glück“, in: Ders., poèmes - poëtra, a.a.O., S. 86, V. 16-18. Diese Verse folgen gleich einem Zitat aus Celans frühem Gedicht „Drüben“. ist.

59. - Vgl. das Faksimile, das dem Band geschriebes. selbst mit stein beigegeben

60. - In der dritten bzw. vierten Strophe: ,und Würfelwurf // hasard, par hasard, schön: / als könnte man winterlang würfeln“ (V. 10-12) und ganz am Ende das Zitat: „,... aussi loin qu'un endroit / fusionne avec au-delà ..."(V. 32f.).

61. - Typoskript, 13. September 1978, Archiv Rainer Blum, Berlin.

62. — Duden, s.v. Kassiber. 
B_Dur Sonate, Schubert, auch so ein Kassiber."63 Diese Passage, die die Art des Empfangens beschreibt, ergänzt auf eigentümliche Weise die erste, die den Herkunftsort des Materials schildert. Zwischen den beiden vollzieht sich eine Verwandlung, die derjenigen im vierzig Jahre später entstandenen Titel geschriebes. selbst mit stein entspricht. Die aufgelesenen Steinchen stehen sinnbildlich für die Widerständigkeit und Überlebensfähigkeit der Schrift, die ein Mensch - ein Steinbruch - von sich abtragen lässt. Ist die Metapher einmal aufgelöst, können die Steinchen zu Kassibern werden. Die Bezeichnung des Geschriebenen als Kassiber deutet zum einen auf den jüdischen Hintergrund dieser Texte hin, zum anderen beschreibt sie ihre bescheidene Kleinteiligkeit, ohne dadurch ihre Wichtigkeit zu schmälern. Die Wahl des dem Jiddischen angelehnten Wortes „geschriebes“ ist davon ein Widerhall. Die Haut des Menschen aber ist dick und lässt von außen wenig herein. Daher besitzen diese Zettel die Härte und Schärfe des Steins, damit sie - in einem Bild, das von Schmerz und Gewalt nicht frei ist - durch die Hautwand und das Gitter der Rippen eindringen (es ist ein freiwilliger Akt, den jeder für sich vollziehen kann) und Teil des Körpers werden können. Dass Mueller ausgerechnet ein musikalisches Beispiel für seine Auffassung der Kassiber wählen sollte, führt zur Musik und zum Lied zurück, die Bestandteil von Muellers eigenen Kassibern sind.

Gedichte sind für Mueller Gelebtes, Erlittenes, Geerbtes, vor allem aber an den (oft gebrechlichen) Körper und an die individuelle Existenz Gebundenes ${ }^{64}$, das auf heimliche, oft geheimnisvolle Weise in Umlauf gerät und im besten Fall Teil des Lebens anderer wird. Die Botschaft, die sie enthalten, ist subversiv, weil sie den Leser auf Schritt und Tritt über die Unebenheiten des Deutschen stolpern lässt. Wie ein Stein des Anstoßes steht sie da, um eingesammelt und mitgenommen zu werden:

Da ist es

: zerschlissen, dieses /

... was

zählst du; du / ich :

um vier Uhr früh. / Früh wird's

abgetschilpt. Es überdauert

dich,

was vom Grün pfeift, von den

Firsten : Antennen

63. - Typoskript, 14. September 1978, Archiv Rainer Blum, Berlin.

64. - Vgl. dazu ausführlich meinen Aufsatz „Mit Nadel \& Faden“(Mütze, 14/2016, S. 678-706) in Bezug auf Muellers Gedicht „Rißvernähung: Oxygène“ (geschriebes, a.a.O., S. 35). 


\author{
„Firstendach“ : Worten / \\ tön ..., so \\ oben ein Schönes ist, \\ ist's Tönen, so. \\ Haben zu sehen, so \\ zu hören. So, zu tönen. \\ Es ist hör'n wie einer / \\ denkt's : Stille65
}

65. - R.R. Mueller, poèmes - poëtra, a.a.O., S. 78. Erstdruck in Park. Zeitschrift für neue Literatur, 24-25/1985, 11. 
